Canadian Oncology

Nursing Journal

Revue canadienne

de soins infirmiers

en oncologie

Volume 27, Issue 1 • Winter 2017

elSSN: $2368-8076$ 


\section{Living with cancer and other chronic conditions: Patients' perceptions of their healthcare experience}

by Kia Duthie, Fay J. Strohschein, and Carmen G. Loiselle

\section{ABSTRACT}

Multimorbidity is known to contribute to the complexity of care for patients with cancer. This qualitative study begins to explore cancer patients' experience with multimodal treatments, that is, treatments for multiple chronic conditions, as well as issues related to navigating the healthcare system. Participants ( $n=10)$ were recruited from an ambulatory cancer centre in a large, university-affiliated hospital in Montreal, Quebec. Important challenges were reported in terms of striking a fine balance between acute health needs and underlying ongoing chronic condition(s), experiencing unforeseen treatment complications, and negotiating silos across medical specialties. Participants also wished to be better known by the healthcare team. When reporting a positive care coordination experience, participants often attributed it to the intervention by a nurse navigator. Lastly, participants expected a more personalized care approach and would have liked to be included in multidisciplinary board meetings. Study results underscore the impetus to better integrate care across diseases, enhance person-centred care, and support patients who strive to balance competing needs when facing multimorbidity.

\section{INTRODUCTION}

$\mathrm{T}$ he number of adults diagnosed with cancer who concurrently have one or more chronic conditions is steadily growing. Aging is associated with a higher incidence of cancer and other age-related chronic conditions such as diabetes, chronic obstructive pulmonary disease, heart disease, arthritis, and hypertension (Canadian Cancer Society, 2012). At least $78 \%$ of older individuals with cancer (aged 60 and older) are also living with one or more chronic conditions (Deckx et al., 2012; Ritchie et al., 2011). Older individuals with three or more chronic conditions have nearly three times the

\section{ABOUT THE AUTHORS}

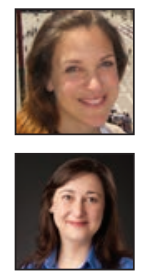

Kia Duthie, RN, MSc, Staff Nurse, BC Cancer Agency, 401 - 122 Walter Hardwick Ave, Vancouver, BC V5Y 0C9

514-726-3902; kia.duthie@gmail.com

Fay J. Strohschein, RN, PhD (candidate), Ingram School of Nursing, McGill University, Nursing Counsellor, Segal Cancer Centre, Jewish General Hospital, 3755 Côte-Sainte-Catherine Rd, Pav. H-304.4, Montreal, QC H3T 1E2

514-340-8222 ext. 3864; fay.strohschein@mail.mcgill.ca

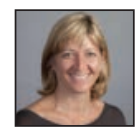

Corresponding Author: Carmen G. Loiselle, N, PhD, Associate Professor, Department of Oncology and Ingram School of Nursing, McGill University, Co-Director (Academic) and Senior Investigator, Segal Cancer Centre, Jewish General Hospital, 3755 Côte-Sainte-Catherine Rd, Pav. E-748, Montreal, QC H3T 1E2

514-340-8222 ext. 3940; carmen.loiselle1@mcgill.ca

DOI: $10.5737 / 236880762714348$ number of healthcare visits than individuals with no chronic conditions (Canadian Institute for Health Information, 2011). Given a growing population living with cancer and co-occurring conditions (Canadian Cancer Society's Advisory Committee on Cancer Statistics, 2015), a better understanding is needed of the healthcare experiences for this complex needs group.

\section{Multimorbidity and the Cancer Trajectory}

Multimorbidity is defined as the coexistence of two or more chronic or acute conditions within one person (Marengoni et al., 2011; van den Akker et al., 1996). Multimorbidity can significantly influence patients' experiences with a chronic illness such as cancer and their interactions with the healthcare system (Smith et al., 2008; Geraci et al., 2005). Several studies have documented that co-occurring diseases often negatively impact the cancer trajectory at every stage including diagnosis, prognosis, treatment, and survival (Extermann, 2007; Louwman et al., 2005; Meyerhardt et al., 2003). Co-occurring conditions can also worsen the patient's cancer treatment response, prognosis, survival rate, and subsequent health outcomes (Extermann, 2007; Meyerhardt et al., 2003; Wolf et al., 2005). Elderly cancer patients are less likely to receive aggressive, curative-intent treatment (Canadian Partnership Against Cancer, 2016a), which may be due, in part, to co-occurring chronic conditions (Chen et al., 2012; Srokowski et al., 2009).

\section{Multimorbidity and Treatment Planning}

Treatment planning is one area of concern when addressing complex interactions between cancer and concurrent illnesses. When an individual with multiple diseases is diagnosed with cancer, treatment decisions must integrate, coordinate, and prioritize treatment modalities (Boyd et al., 2005; Yancik et al., 1998). For example, the healthcare team needs to be mindful of possible medication interactions during the cancer treatment process as current clinical practice guidelines only focus on one illness at a time (Lee et al., 2011; Lindenfeld \& Kelly, 2010; Tinetti et al., 2004). Failing to address complex patients' needs may result in undesirable outcomes (Boyd et al., 2005). Due to the limited availability of resources and standards for healthcare teams, there is an increased risk of unforeseen treatment complications and suboptimal quality of care and life for these patients.

\section{Multimorbidity and Challenges with Care Coordination}

Challenges in incorporating cancer treatment plans into the primary care of chronic conditions relates to the involvement of multiple clinicians, potential medication interactions, side effects, and competing healthcare needs. Optimal health management requires frequent communication among clinicians, patients, and family members. Unfortunately, efficient 
Table 1: Participant Characteristics

\begin{tabular}{|l|l|l|l|}
\hline Age & Gender & Cancer Types & Chronic Conditions \\
\hline 62 & F & Breast cancer, colorectal cancer & Autoimmune myesthenia gravis \\
\hline 55 & F & Endometrial cancer & Diabetes \\
\hline 68 & M & B cell lymphoma & Diabetes, hypertension, high cholesterol, haemochromatosis (requiring Coumadin) \\
\hline 62 & M & Prostate cancer & Chronic depression \\
\hline 67 & M & Colorectal cancer & Hypertension, diabetes, pacemaker (requiring Coumadin) \\
\hline 52 & F & Colorectal cancer & Diabetes, high cholesterol \\
\hline 71 & F & Breast cancer & Peripheral neuropathy, osteoarthritis, basal cell carcinomas \\
\hline 79 & M & Multiple Myeloma & Glaucoma, hypertension, high cholesterol \\
\hline 70 & M & Chronic lymphocytic leukemia & Hyperthyroidism, hypertension, high cholesterol \\
\hline 73 & F & Follicular and B cell lymphoma & Cutaneous lupus, Sjogrens syndrome \\
\hline
\end{tabular}

coordination of services and communication often do not occur, resulting in unmet patient needs and negative health outcomes (Burgers et al., 2010; Jabaaij et al., 2012). Studies indicate that individuals with a higher number of chronic conditions report less favourable experiences with healthcare providers (Bowker et al., 2006; Burgers et al., 2010).

Several studies address cancer patients' perceptions of care and services (Sitzia \& Wood, 1998; van Der, 1999; Saultz \& Albedaiwi, 2004; Puts et al., 2012), but few studies have thoroughly explored the needs of patients with cancer and co-occurring conditions. This qualitative study was designed to obtain rich descriptions from the patient perspective (Magilvy $\&$ Thomas, 2009) of their experience with multimodal treatments, often complex healthcare needs, and navigating the healthcare system. While striving to maintain an exploratory approach that allowed for the emergence of participant concerns and perspectives, the literature suggesting the complexity associated with multimorbidity, outlined above, as well as the values associated with person-centred care (Kitson, et al., 2013; Lusk \& Fater, 2013) formed the conceptual frame for this study. By identifying common themes associated with multimorbidity, the study results may inform current clinical practice and how healthcare teams could better meet the complex needs of this specific patient population.

\section{METHODS}

\section{Participants}

Patients diagnosed with cancer and at least one co-occurring chronic condition were approached at an ambulatory cancer centre in a large, university-affiliated hospital in Montréal, Québec. This ambulatory cancer centre provides comprehensive cancer care including cancer screening, diagnosis, treatment, pain and symptom management, palliative care, and psychosocial support. Nurse navigators in the oncology departments recruited participants who met the following criteria: 50 years of age or older, English-speaking, diagnosed with cancer and at least one co-occurring disorder, and had undergone cancer treatment (chemotherapy, radiation, and/or surgery) within the last 12 months. Exclusion criteria included any serious pre-existing physical or mental health condition that would affect the participant's ability to complete the study. Ten of the 14 patients who were approached agreed to participate (4 patients declined due to self-reported time constraints). The enrolled participants ( 5 females and 5 males) were between the ages of 52 and $79(\mu=66)$ and self-reported cancer diagnoses included: colorectal, breast, B cell lymphoma, endometrial, prostate, multiple myeloma, and chronic lymphocytic leukemia (Table 1).

\section{Interview Protocol}

Participants were interviewed individually for 40 to $80 \mathrm{~min}$ utes at the hospital, in a private location, or in the participant's home. The semi-structured interview format included openended questions such as, "Can you tell me about your experience with your cancer treatment?", "Can you tell me about other health conditions or concerns you have been diagnosed with?", and "What do you do to take care of this/these condition(s)?" Socio-demographic information was collected using a self-report questionnaire.

The interviews were digitally audio-recorded, transcribed verbatim and analyzed using applied thematic analysis, an exploratory approach that focuses on the description and understanding of people's feelings, thoughts, and behaviours within a specific context (Guest et al., 2011). It integrates key methodological techniques to offer "a rigorous, yet inductive set of procedures designed to identify and examine themes from textual data" (p. 15, Guest et al., 2011). Central to this approach is open coding of transcripts to reduce data to short descriptions or phrases and identify key concepts. Related descriptions or phrases are then grouped together into categories, studied for patterns of similar concepts and meaning, and regrouped into key themes (Magilvy \& Thomas, 2009; Guest et al., 2011). To ensure data trustworthiness (Lincoln \& Guba, 1985; Polit \& Beck, 2010; Tuckett, 2005; Koch, 2006), the first author discussed the emerging analysis with peers and academic supervisors throughout data collection and 
analysis, integrated emerging analyses into subsequent interviews to check analytic concepts with participants, and kept an audit trail that included all audio-recordings, transcripts, and detailed records of the research process including the steps involved in collecting and analyzing data.

\section{RESULTS}

Data analysis revealed several key themes. The most salient was related to participants' need to establish a 'fine balance' between the demands of their cancer treatment and those of their other chronic conditions. Participants who reported more equilibrium between cancer treatment demands and other chronic condition management described their experience as a 'holistic, personalized, and caring approach.' Whereas participants who reported negative experiences expressed concerns over 'not feeling known or heard by the healthcare team.' Participants in both groups also spontaneously identified the nurse navigator (a healthcare provider who accompanies and supports patients and families throughout the trajectory of the cancer experience) as an essential positive factor in the quality of their care and their experience of managing multimorbidities. An additional theme emerged around participants' expressed need to be present during multidisciplinary meetings, also called medical rounds or tumour board meetings.

\section{Striking a Fine Balance: Concrete challenges associated with multimorbidity}

Data analysis suggested that participants' ability to manage cancer treatment was more difficult in the presence of certain chronic conditions, including diabetes and those requiring medication such as Coumadin. Participants with diabetes, for instance, reported salient challenges including self-care issues, medication interactions, painful and worrying side effects, care and/or medication errors, financial concerns, and poor care coordination. These participants reported challenges in diabetes management due to cancer treatment. For example, a participant who was prescribed prednisone as part of cancer treatment struggled with elevated blood glucose levels. Participants also reported challenges with managing Coumadin dosages as a result of cancer treatment: "Now everything is upside down because of the chemotherapy." One participant reported side effects from increased dosages of Coumadin such as delayed clotting and healing and another participant reported significant financial concerns when he was forced to switch medications due to Coumadin incompatibility with his cancer treatment, stating that the new medication was "extremely expensive" and a major source of stress for him.

Participants with other chronic conditions such as cutaneous lupus, hypertension, hypercholesterolemia, hyperthyroidism, depression, and osteoarthritis reported that cancer treatment did not have a significant impact on the management of their chronic condition. They described a relatively steady balance and felt able to focus on their cancer treatment as the new priority: “I take my other medication, like I have to. But I don't focus on that much because this [cancer] supersedes it all." They also described, in general, positive experiences with the healthcare system, with most of their needs being met. Three participants reported, "The nurses, the way the system is working here, it's excellent," "The care and the coordination of the treatment I've gotten have been unbelievably good," and "I think I'm being well, very well taken care of."

\section{Not Feeling Known or Heard by the Healthcare Team}

In addition to challenges for some to balance the demands from multimorbidities, participants with health-related unmet needs described obvious gaps in care despite their requests for additional support. They expressed concerns of "not feeling known" and "not feeling heard" by the healthcare team. These participants felt that their healthcare providers did not thoroughly read their medical file or have a good grasp of their overall medical situation: "I had the feeling at times that they don't have all the information, that many people who did look at my file didn't read the file carefully." One participant reported being frequently asked the same questions and providing the same medical information "all over again." $\mathrm{He}$ expressed being frustrated about this: "You [the provider] have this bloody record, you should go into the records and check!"

Participants reported a desire for clearer communication and engagement around treatment planning and acknowledgement of their healthcare needs. One participant felt surprised and grateful about a particular surgeon who took the time to thoroughly explain the treatment plan to him, saying, "He is the [only one] over six to seven years of my life that took so much time explaining." This participant discussed how important it was to take time with patients to get to know them and help them feel at ease: "You are dealing with people, and dealing with people takes time." He felt that taking this time to address patients' concerns was "very unique to certain people who really, really care." This illustrates the importance of improving provider-patient communication.

When clear explanations and information were not provided, participants felt anxious, confused, and stressed, mostly as a result of not understanding certain treatment decisions and processes. One participant was informed that her cancer was aggressive and fast growing, yet had to wait to start chemotherapy and she wondered, "How can you wait like that [to start treatment]?" She also mentioned not understanding why she had not yet gone through surgery, saying, "I'm a little nervous and upset about these things."

\section{A Comprehensive Personalized Care Approach}

Participants who did not experience complications with management of their chronic conditions reported appreciating a 'holistic personalized caring approach' from the healthcare team that addressed their concerns: "I was told, 'If you have any situation, financial situation or so, you should let us know about it, it's something we can help you with" and "They don't want you to have anything to worry about." These participants also mention valuing the time healthcare providers took to tailor the care to patients' individual needs: "He gets to know his patients, and knows how to give you just the amount of information you can handle." They also mentioned being made to feel very secure and comfortable by members of the team: "When I first walked in here, I was the [most scared] person in the world. And now I feel so comfortable, because of you people [the healthcare team]." 


\section{The Nurse Navigator as Essential Component of Quality Care}

Participants identified the nurse navigator in oncology as one of the most important resources to go to for information, emotional support, and improved continuity of care while going through the complexities of cancer treatment. Participants often expressed their appreciation for the nurse navigator: "It's a saviour, to have a system like that. Any issue that I've had, any question, I'm home and I'm concerned or whatever, I call her. And she calls back," "She knows everything, she's there for everything. I think I talk to her more than I talk to my doctor. I am more comfortable with her", and "She has lots of answers clearly for me." These quotes highlight the central role the nurse navigator played. Participants also described valuing the nurse's role in facilitating communication amongst healthcare team members as it related to their care: "She's my liaison", "[She is] intervening, making sure all the people treating me are talking together," and "You need somebody who can talk to everybody."

\section{Being Included in Multidisciplinary Meetings}

A salient desire expressed by participants was to be invited to meetings with the entire healthcare team, including nurses, oncologists, surgeons, and even the hospital ombudswoman. They described wanting an opportunity to share with the team their personal experiences and challenges: "This is how I react, this is my problem, this is how my body reacts, this is how my intellect reacts, this is how my emotional state is reacting." Participants shared that they also wanted the opportunity to increase their health knowledge by asking questions to the whole healthcare team and be more involved in the treatment planning process. The possibility of participating in multidisciplinary meetings was also described as enhancing patients' feelings of being heard and known. One participant reported not being consulted during an extensive assessment and evaluation by the team, stating, "I know myself better than anybody... I know how my body reacts to certain things." Another mentioned feeling frustrated about not being invited to contribute to his treatment plan and care decisions, when "in the end it's my life ... I know myself, I know what's best." This underscores the need for the healthcare team to recognize the importance and value of involving patients in making treatment decisions and developing care plans.

\section{DISCUSSION}

In exploring healthcare experiences of patients with cancer and other chronic conditions, key aspects related to striking a balance between chronic and more acute healthcare needs, as well as feeling better known and heard by the healthcare team. The latter issue is actively being addressed through a strong movement towards person-centred care (Canadian Partnership Against Cancer, 2016b) - an individualized and holistic care approach that is customized to an individual's unique needs, values, and perspectives (Kitson, et al., 2013; Lusk \& Fater, 2013). Participants described valuing or desiring an approach from the team that focuses on their personal needs and preferences. Despite recent efforts to shift the focus of cancer care away from the traditional paternalistic approach towards a more person-centred approach, studies suggest that person-centred care continues to be a challenge particularly for patients with multiple medical conditions (Bayliss et al., 2008; Fashoyin-Aje et al., 2012). It is vital to systematically include patients in defining and operationalizing what person-centred care means to them, in order to improve support and overall quality of care.

Corroborating our findings, other studies have found that participants with multiple conditions report problematic interactions with the healthcare team and feelings of being neglected (Noël et al., 2005), as well as unmet health needs and lack of comprehensive medical care due to time constraints during provider consultations (Clarke et al., 2013). Another study identified clear communication, individualized care plans, and healthcare provider acknowledgement of patient needs as care processes strongly desired by patients with multiple conditions (Bayliss et al., 2008). This goal could be accomplished by providers being more acquainted with patients' medical history, having a good understanding of the whole medical picture before meeting with the patient, and being aware of patients' individual preferences for cancer information (Lambert, Loiselle, \& Macdonald, 2009).

In addition, clear provider-patient communication regarding treatment decisions, potential side effects, and possible drug interactions is essential. Healthcare providers could also periodically verify with patients if gaps in care coordination exist to reduce the risk of unmet needs or complications (Extermann, 2007; Louwman et al., 2005; Meyerhardt et al., 2003). Poor care coordination may result in patients being asked repeatedly for the same medical information, and neglecting the next steps in care, it seems key to rephrase certain healthcare questions and subsequently validate with patients such as "I saw in your chart that... is this still the case for you?" (Clausen et al, 2012). Care providers are thus offering reassurance to the patient that the care provider is aware of the information in the chart while also inviting the patient's input and perspective on their current health concerns.

Participants also expressed a desire to be included in multidisciplinary discussions, which may address communication gaps, as well as the need to be better known to the healthcare team, as identified above. There is limited literature on including patients in these meetings, but the important role for patient participation in developing interdisciplinary care plans has been identified (Clausen, et al., 2012). Also, research undertaken on family-centred rounds at the bedside (or more recently on nursing rounds) found that involving patients improved communication between families and the healthcare team and correlated with increased quality of care ratings and patient satisfaction, as well as decreased anxiety (Delgado et al., 2009). Despite efforts to promote patient involvement in healthcare delivery, studies are often lacking attention to what particular roles patients wish to play (Thompson, 2007). Our findings identify one clear participant preference, which is to increase involvement in healthcare team venues or meetings.

A promising strategy to improve communication and care coordination is to involve nurse navigators (also called pivot nurses in Québec) across phases of the cancer/multimorbidity 
healthcare experience. The nurse navigator accompanies patients with complex needs through the cancer journey, assessing and managing patient and family needs, providing information and education, providing support, and helping coordinate care and services (De Serres \& Beauchesne, 2000; Horner et al., 2013). Participants identified the nurse navigator as an essential resource for emotional support, information needs, and continuity of care while navigating the complexities of the healthcare system. Patients whose chronic condition management became more complicated specifically mentioned appreciating the role of the nurse navigator in helping facilitate communication among providers, and having this one 'point of focus' or 'hub' at the center of the healthcare wheel.

It is important to consider that participants herein were recruited by nurse navigators, so they may have a closer relationship with these nurses, thus holding more positive views or feeling the need to provide positive feedback (demand characteristics). However, Bayliss et al. (2008) also found patients with multiple conditions desired "support from a single coordinator of care who could help patients prioritize the competing demands from their multiple conditions and continuity of relationships" (p. 289). Other studies have found that oncology nurse navigators help patients manage their symptoms and other conditions, reduce patients' cancer-related distress, and increase patient satisfaction and overall quality of life ratings (Swanson \& Koch, 2010; Jennings-Sanders \& Anderson, 2003). Nurse navigators should be encouraged to also communicate

\section{REFERENCES}

Bayliss, E.A., Edwards, A.E., Steiner, J.F., \& Main, D.S. (2008). Processes of care desired by elderly patients with multimorbidities. Family Practice, 25, 287-293.

Bowker, S.L., Pohar, S.L., \& Johnson, J.A. (2006). A cross-sectional study of health-related quality of life deficits in individuals with comorbid diabetes and cancer. Health and Quality of Life Outcomes, $4,1$.

Boyd, C.M., Darer, J., Boult, C., Fried, L.P., Boult, L., \& Wu, A.W. (2005). Clinical practice guidelines and quality of care for older patients with multiple comorbid diseases: Implications for pay for performance. JAMA, 294, 716-724.

Brandenbarg, D., Roorda, C., Groenhof, F., Havenga, K., Berger, M.Y., de Bock, G.H., \& Berendsen, A.J. (2014). Increased primary healthcare use in the first year after colorectal cancer diagnosis. Scandinavian Journal of Primary Healthcare, 32, 55-61.

Burgers, J.S., Voerman, G.E., Grol, R., Faber, M.J., \& Schneider, E.C. (2010). Quality and coordination of care for patients with multiple conditions: Results from an international survey of patient experience. Evaluation a the Health Professions, 33, 343-364.

Canadian Cancer Society. (2012). General cancer statistics at a glance. Retrieved from https://www.cancer.ca/Canadawide/About\%20 cancer/Cancer\%20 statistics / Stats\%20at\%20a\%20glance/ General\%20cancer\%20stats.aspx?sc_lang=EN.

Canadian Cancer Society's Advisory Committee on Cancer Statistics. (2015). Canadian Cancer Statistics 2015. Retrieved from https:// www.cancer.ca/ /media/cancer.ca/CW/cancer\%20information/ cancer\%20101/Canadian\%20cancer\%20statistics/CanadianCancer-Statistics-2015-EN.pdf regularly with the patient's general practitioner, who often plays an active role in managing patients' other conditions throughout cancer treatment (Klabunde et al., 2009). Patients with cancer have $54 \%$ more visits and $68 \%$ more drug prescriptions by their general practitioner in the year following a cancer diagnosis (Brandenbarg et al., 2014).

The burden associated with a cancer diagnosis is often significant, with medical care becoming increasingly complex and often involving numerous healthcare specialties. These challenges are magnified when patients also deal with additional chronic health conditions. In this context, meeting informational, psychosocial, physical and coordination needs is paramount. These findings highlight important aspects of person-centred care from patients' perspectives and suggest those with multiple conditions have additional complex needs for treatment planning and care coordination. These findings also provide further evidence of the immense value of the nurse navigator role in oncology. This role should continue to be developed and supported to provide optimal care for cancer patients with complex care situations such as multiple comorbidities. Lastly, these results suggest that patients desire being involved in multidisciplinary meetings to help come to a shared consensus in complex cases or when there are differing goals and priorities with regards to the care plan. The provision of a personalized and comprehensive approach for patients experiencing complex illness management is necessary to optimize health-related outcomes and enhance the patient experience with healthcare.

Canadian Institute for Health Information. (2011). Seniors and the Healthcare System: What is the Impact of Multiple Chronic Conditions? Retrieved from https://secure.cihi.ca/free_products/ air-chronic_disease_aib_en.pdf

Canadian Partnership Against Cancer. (2016a). The 2016 Cancer System Performance Report. Retrieved from http://www. systemperformance.ca/reports/

Canadian Partnership Against Cancer. (2016b). We see progress: The Canadian strategy for cancer control, 2017-2022. Retrieved from http://www.partnershipagainstcancer.ca/wp-content/uploads/ sites/5/2016/02/canadian-strategy-cancer-control-2017-2022-en.pdf

Chen, R. C., Royce, T. J., Extermann, M., \& Reeve, B. B. (2012). Impact of age and comorbidity on treatment and outcomes in elderly cancer patients. Seminars in Radiation Oncology, 22, 265-71.

Clarke, L.H., Bennett, E.V., \& Korotchenko, A. (2013). Negotiating vulnerabilities: How older adults with multiple chronic conditions interact with physicians. Canadian Journal on Aging/La Revue canadienne du vieillissement, 33, 1-12.

Clausen, C., Strohschein, F., Faremo, S., Bateman, D., Posel, N., \& Fleiszer, D. (2012). Developing an interprofessional care plan for an older adult woman with breast cancer: From multiple voices to a shared vision. Clinical Journal of Oncology Nursing, 16(1), E18E25. doi:10.1188/12.CJON.E18-E25

De Serres, M., \& Beauchesne, N. (2000). L'intervenant pivot en oncologie: un rôle d'évaluation, d'information et de soutien pour le mieux-être des personnes atteintes de cancer: document de réflexion, [Québec]: Conseil québécois de lutte contre le cancer. 
Deckx, L., van den Akker, M., Metsemakers, J., Knottnerus, A., Schellevis, F., \& Buntinx, F. (2012) Chronic diseases among older cancer survivors. Journal of Cancer Epidemiology, 2012, 1-7.

Delgado, E.M., Callahan, A., Paganelli, G., Reville, B., Parks, S.M., \& Marik, P.E. (2009). Multidisciplinary family meetings in the ICU facilitate end-of-life decision making. American Journal of Hospice and Palliative Medicine, 26, 295-302.

Extermann, M. (2007). Interaction between comorbidity and cancer. Cancer Control, 14, 13-22.

Fashoyin-Aje, L.A., Martinez, K.A., \& Dy, S.M. (2012). New patientcentered care standards from the Commission on Cancer: Opportunities and challenges. The Journal of Supportive Oncology, 10, 107-111.

Geraci, J.M., Escalante, C.P., Freeman, J.L., \& Goodwin, J.S. (2005). Comorbid disease and cancer: The need for more relevant conceptual models in health services research. Journal of Clinical Oncology, 23, 7399-404.

Guest, G., Macqueen, K.M., \& Namey, E.E. (2011). Applied thematic analysis. Sage.

Horner, K., Ludman, E.J., Mccorkle, R., Canfield, E., Flaherty, L., Min, J., ... Wagner, E.H. (2013). An oncology nurse navigator program designed to eliminate gaps in early cancer care. Clinical Journal of Oncology Nursing, 17, 43-48.

Jabaaij, L., Van Den Akker, M., \& Schellevis, F. (2012). Excess of healthcare use in general practice and of comorbid chronic conditions in cancer patients compared to controls. BMC Family Practice, 13, 60.

Jennings-Sanders, A., \& Anderson, E.T. (2003). Older women with breast cancer: perceptions of the effectiveness of nurse case managers. Nursing Outlook, 51, 108-114.

Kitson, A., Marshall, A., Bassett, K., \& Zeitz, K. (2013). What are the core elements of patient-centred care? A narrative review and synthesis of the literature from health policy, medicine and nursing. Journal of Advanced Nursing, 69(1), 4-15. doi:10.1111/j.1365-2648.2012.06064.x

Klabunde, C.N., Ambs, A., Keating, N.L., He, Y., Doucette, W.R., Tisnado, D., ... Kahn, K.L. (2009). The role of primary care physicians in cancer care. Journal of General Internal Medicine, 24(9), 1029-1036. doi:10.1007/s11606-009-1058-x

Koch, T. (2006). Establishing rigour in qualitative research: The decision trail. 1993. Journal of Advanced Nursing, 53, 91-100; discussion 101-3.

Lambert, S.D., Loiselle, C.G., \& Macdonald, M.E. (2009). An in-depth exploration of information-seeking behavior among individuals with cancer: Part 1 Understanding differential patterns of active information-seeking. Cancer Nursing, 32, 11-25.

Lee, L., Cheung, W.Y., Atkinson, E., \& Krzyzanowska, M.K. (2011). Impact of comorbidity on chemotherapy use and outcomes in solid tumors: A systematic review. Journal of Clinical Oncology, 29, 106-117.

Lincoln, Y.S., \& Guba, E.G. (1985). Naturalistic inquiry. Beverly Hills, CA: Sage Publications.

Lindenfeld, J., \& Kelly, P.A. (2010). Developing a cardiology-oncology clinical practice guideline. Progress in Cardiovascular Diseases, 53, 173-9.

Louwman, W.J., Janssen-Heijnen, M.L.G., Houterman, S., Voogd, A.C., van der Sangen, M.J.C., Nieuwenhuijzen, G.A.P., \& Coebergh, J.W.W. (2005). Less extensive treatment and inferior prognosis for breast cancer patient with comorbidity: A populationbased study. European Journal of Cancer, 41, 779-785.

Lusk, J.M., \& Fater, K. (2013). A concept analysis of patient-centered care. Nursing Forum, 48(2), 89-98. doi:10.1111/nuf.12019
Magilvy, J.K., \& Thomas, E. (2009). A first qualitative project: qualitative descriptive design for novice researchers. Journal for Specialists in Pediatric Nursing, 14, 298-300.

Marengoni, A., Angleman, S., Melis, R., Mangialasche, F., Karp, A., Garmen, A., ... Fratiglioni, L. (2011). Aging with multimorbidity: A systematic review of the literature. Ageing Research Reviews, 10, 430-439.

Meyerhardt, J.A., Catalano, P.J., Haller, D.G., Mayer, R.J., Macdonald, J.S., Benson, A.B., 3rd, \& Fuchs, C.S. (2003). Impact of diabetes mellitus on outcomes in patients with colon cancer. Journal of Clinical Oncology, 21, 433-40.

Noël, P.H., Chris, F.B., Larme, A.C. \& Pugh, J.A. (2005). Collaborative care needs and preferences of primary care patients with multimorbidity. Health Expectations, 8, 54-63.

Polit, D.F., \& Beck, C.T. (2010). Essentials of nursing research: Appraising evidence for nursing practice. Lippincott Williams \& Wilkins.

Puts, M.T.E., Papoutsis, A., Springall, E., \& Tourangeau, A.E. (2012). A systematic review of unmet needs of newly diagnosed older cancer patients undergoing active cancer treatment. Supportive Care in Cancer, 20, 1377-1394.

Ritchie, C.S., Kvale, E., \& Fisch, M.J. (2011). Multimorbidity: an issue of growing importance for oncologists. Journal of Oncology Practice, 7(6), 371-374.

Rodgers, B.L., \& Cowles, K.V. (1993). The qualitative research audit trail: A complex collection of documentation. Research in Nursing Q Health, 16, 219-26.

Saultz, J.W., \& Albedaiwi, W. (2004). Interpersonal continuity of care and patient satisfaction: A critical review. The Annals of Family Medicine, 2, 445-51.

Sitzia, J., \& Wood, N. (1998). Study of patient satisfaction with chemotherapy nursing care. European Journal of Oncology Nursing, 2, 142-153.

Smith, A.W., Reeve, B.B., Bellizzi, K.M., Harlan, L.C., Klabunde, C.N., Amsellem, M., ... Hays, R.D. (2008). Cancer, comorbidities, and health-related quality of life of older adults. Healthcare Finance Review, 29, 41-56.

Srokowski, T.P., Fang, S., Hortobagyi, G.N., \& Giordano, S.H. (2009). Impact of diabetes mellitus on complications and outcomes of adjuvant chemotherapy in older patients with breast cancer. Journal of Clinical Oncology, 27, 2170-2176.

Swanson, J., \& Koch, L. (2010). The role of the oncology nurse navigator in distress management of adult inpatients with cancer: A retrospective study. Oncology Nursing Forum, 37, 69-76.

Thompson, A. (2007). The meaning of patient involvement and participation in healthcare consultations: a taxonomy. Social Science Q Medicine, 64, 1297-1310.

Tinetti, M.E., Bogardus, S.T., Jr., \& Agostini, J.V. (2004). Potential pitfalls of disease-specific guidelines for patients with multiple conditions. The New England Journal of Medicine, 351, 2870-4.

Tuckett, A.G. (2005). Part II. Rigour in qualitative research: complexities and solutions. Nurse Researcher, 13, 29-42.

van Der, M. (1999). Relating information needs to the cancer experience: 1. Information as a key coping strategy. European Journal of Cancer Care, 8, 238-244.

van den Akker, M., Buntinx, F., \& Knottnerus. (1996). Comorbidity or multimorbidity. European Journal of Clinical Practice, 2, 65-70.

Wolf, I., Sadetzki, S., Catane, R., Karasik, A., \& Kaufman, B. (2005). Diabetes mellitus and breast cancer. The Lancet Oncology, 6, 103-111.

Yancik, R., Wesley, M.N., Ries, L.A., Havlik, R.J., Long, S., Edwards, B.K., \& Yates, J.W. (1998). Comorbidity and age as predictors of risk for early mortality of male and female colon carcinoma patients: A population-based study. Cancer, 82, 2123-34. 